\title{
Salud y Seguridad del Trabajo en Portugal: apuntes diversos
}

\author{
Some annotations about Occupational Health \\ and Safety in Portugal
}

António de Sousa Uva

Médico del Trabajo e profesor catedrático de Salud Ocupacional. Escola Nacional de Saúde Pública - Universidade Nova de Lisboa.

Correspondencia:

António de Sousa Uva

GD de Saúde Ambiental e Ocupacional

Escola Nacional de Saúde Pública/UNL

asuva@ensp.unl.pt

Telef: 217512100 Fax 217582754

Resumen

El autor aborda algunos aspectos relativos a la Salud y Seguridad del Trabajo (SST) en Portugal llamando la atención para, esencialmente, intentar sostener la tesis de que, desde mediados de la década de los 90, se observaron importantes avances en la mejoría de las condiciones del trabajo en la perspectiva de la SST, aunque se mantuvieron algunos problemas en la práctica concreta de las normativas legales.

Incluso con estas mejoras, las tasas de frecuencia de accidentes de trabajo y de enfermedades profesionales en Portugal todavía se mantienen muy elevadas necesitando, por tanto, estrategias más "agresivas" para concienciar la sociedad portuguesa en torno a las políticas públicas de SST efectivamente promotoras de prevención de riesgos profesionales y de la promoción de la salud de quien trabaja.

Es por tanto indispensable que la protección de la salud y de la seguridad de los trabajadores en los puestos de trabajo sea encarada como un conjunto de actividades de indiscutible utilidad para todos los representantes del mundo del trabajo e que la agenda política pase a valorizar, aún más, la promoción de espacios de trabajo saludables y seguros, con la consecuente valorización de las variables individuales en la estrategia nacional de salud y seguridad.

Palabras clave: Portugal, Salud y Seguridad del Trabajo, Accidentes de Trabajo, Enfermedades Profesionales. Med Segur Trab 2009; 55 (214): 12-25

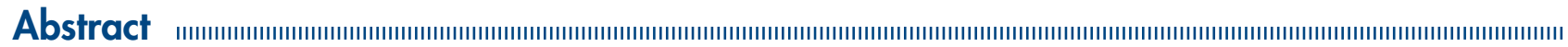

In the present work, the author analyzes several aspects of Occupational Health and Safety (OHS) in Portugal. He provides evidence sustaining the assumption of an important progress, since the mid-nineties, in the improvement of the working conditions from an OHS perspective. Nevertheless, several problems still remain in the practical application of legal regulations.

In addition, despite of these improvements, the frequency rates of working accidents and occupational diseases remain very high. Hence, more "aggressive" strategies are required to increase the awareness of the Portuguese society relative to the OHS public policies, whose effectiveness has been demonstrated either in terms of occupational risks prevention and workers health promotion.

The protection of workers' health and safety at the workplace must then be assumed as an activity of indisputable utility by all stakeholders. The political agenda must thus promote even more the promotion of 
healthy and safe workplaces. As a consequence, there would be also a higher development of individual aspects, and not only environmental data, in the national strategy of occupational health and safety.

Keywords: Portugal: Occupational Health and Safety; Occupational Accidents; Occupational Diseases. Med Segur Trab 2009; 55 (214): 12-25 


\section{INTRODUCCIÓN}

En el transcurso de los últimos dos siglos, los aspectos de la Salud y la Seguridad del Trabajo tienen sido, sistemáticamente, influenciados por innumerables factores económicos, políticos y sociales entre los cuales se destacan, entre otros, la estructura macroeconómica, el nivel de industrialización, la organización (y volumen) de las empresas y el grado de desenvolvimiento (socio-económico y cultural). El avance económico más importante en Portugal ocurrió, históricamente, con la industrialización que se prolongó hasta la llegada del Estado Nuevo (años 30). Hasta el siglo XIX el desarrollo fue lento, disperso, y se redujo esencialmente a la importación de maquinaria de la revolución industrial inglesa y francesa, épocas en las que se realizaron en Portugal obras públicas de grande envergadura ${ }^{1}$.

La influencia negativa de los factores profesionales de la Salud y Seguridad de los trabajadores (SST) envolvió, históricamente, la insalubridad del ambiente de trabajo como factor determinante de la aparición de enfermedades profesionales y de accidentes de trabajo. Las malas condiciones de trabajo, en la perspectiva de la Salud y de la Seguridad, llevaron a la creación de programas de prevención de los riesgos profesionales centrados, por un lado, en el ambiente de trabajo y, por otro lado, en la protección de la salud de los trabajadores expuestos.

A pesar de esto, con el transcurso del tiempo, el desarrollo de las políticas de Salud y Seguridad en el Trabajo no tenían aun, en el contexto de las políticas sociales, la importancia que ciertamente tal tipo de intervención debería adquirir en el mundo del trabajo y se concretizó en principio, en Portugal, en acciones fundamentalmente centradas en la vigilancia médica de los trabajadores, como ya había sucedido en la mayoría de los países mediterráneos.

\section{ASPECTOS GENÉRICOS DE LA ESTRUCTURA EMPRESARIAL PORTUGUESA}

La estructura de las empresas portuguesas tiene actualmente un importante predominio del sector terciario (área de servicios) que, en 2005, ya representaba 78\% del total de las empresas, empleando cerca de $62 \%$ de la población laboral ${ }^{2}$.

La realidad portuguesa, con una población activa de cerca de cinco millones de

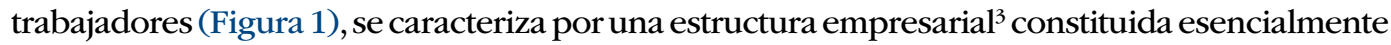
por pequeñas, o incluso muy pequeñas empresas (Tabla I y Figura 2), y por la existencia de un número elevado de trabajadores autónomos y de empresas de naturaleza familiar, lo que condiciona una situación particular en materia de Salud, Higiene y Seguridad en el Trabajo (SH\&ST), donde los modelos organizativos de prestación de cuidados de Salud y Seguridad del Trabajo no siempre tienen auxiliado de forma satisfactoria.

Figura 1 - Portugal: población activa empleada.

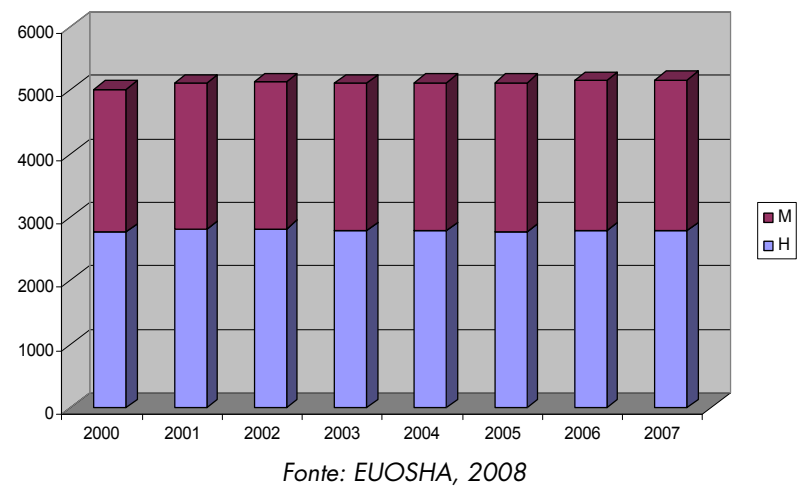

Las empresas con menos de diez trabajadores representaban, en 1997, 80.5\% del total de empresas, aun empleando solamente el $24.7 \%$ de la población activa empleada. 
Datos más recientes del Instituto Nacional de Estadística (INE), relativos al 2004, indicaban que el tamaño medio de las empresas portuguesas era de 9.4 trabajadores ${ }^{4}$.

Tabla I - Portugal: tamaño de las empresas.

\begin{tabular}{|c|c|c|c|c|}
\hline & 1988 & 1990 & 1992 & 1997 \\
\hline hasta 9 trab. . . . . . . . . . . . . . . . & 74,5 & 75,1 & 76,6 & 80,5 \\
\hline 10 a 19 & 12,7 & 12,3 & 11,8 & 10,0 \\
\hline 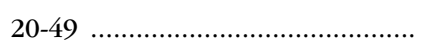 & 8,1 & 7,9 & 7,4 & 6,1 \\
\hline 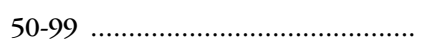 & 2,6 & 2 & 2,4 & 1,8 \\
\hline 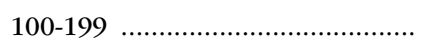 & 1,2 & 1,1 & 1 & 0,8 \\
\hline$\geq 500$ & 0,3 & 0,3 & 0,2 & 0,1 \\
\hline
\end{tabular}

Figura 2 - Portugal: tamaño de las empresas en número de trabajadores.

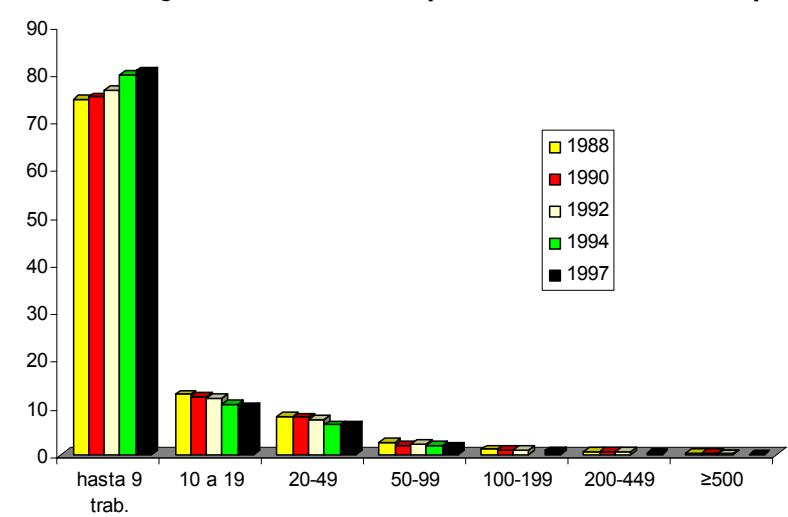

La estructura empresarial portuguesa se caracteriza por un predominio de microempresas ( $<10$ trabajadores). Las microempresas representan, como se señaló, la gran mayoría de las empresas. Este crecimiento (Figura 3) se tiene observado al mismo tiempo que se evidencia un decrecimiento de las empresas de mayor tamaño (pequeñas, medianas y grandes empresas).

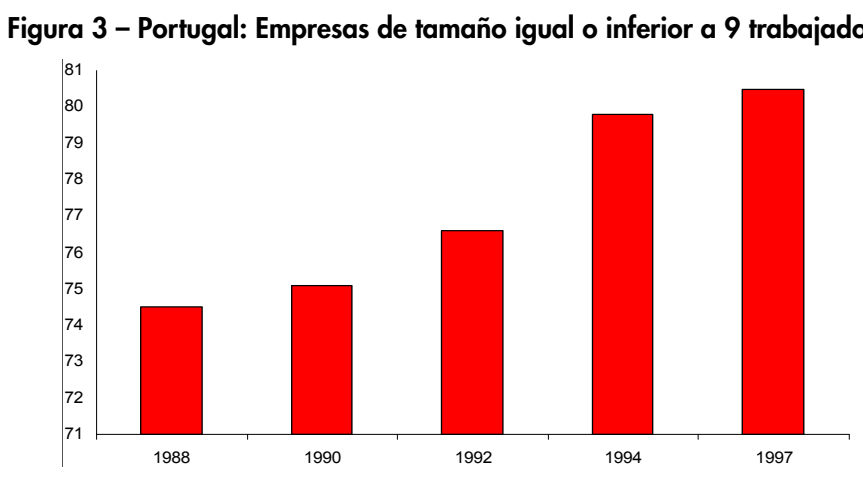

\section{TÉCNICOS DE SALUD Y SEGURIDAD DEL TRABAJO}

Otro aspecto importante relativo a la SST se relaciona con los recursos humanos disponibles. En Portugal, apenas existe alguna información organizada en relación a los médicos del trabajo y los técnicos en Higiene y Seguridad (ahora denominados de Seguridad e Higiene), la mayoría de ellos de nivel V, es decir, con una formación de nivel superior. La pagina Web del Instituto para la Seguridad, Higiene y Salud en el Trabajo de Portugal refería la existencia, a 19 de septiembre del 2005, de 5115 técnicos 
de nivel V y de 1646 de nivel III $^{5}$ y más recientemente teníamos números superiores de, respectivamente, 8140 y $1843^{6}$. Se trata de un importante número de técnicos de Seguridad e Higiene.

En el área médica, la formación específica en Medicina del Trabajo data de la década de los 60. De hecho, la formación en Medicina del Trabajo en Portugal está cerca de completar 50 años de historia ${ }^{7}$. Se inicio en 1963, en el Instituto de Higiene Dr. Ricardo Jorge (en Lisboa), como especialidad del Curso de Medicina Sanitaria y luego a seguir continúo en la Escuela Nacional de Salud Publica (Lisboa). Su inicio está asociado, en términos históricos, a la obligación legal de este título para el ejercicio de la Medicina del Trabajo en servicios médicos del trabajo ${ }^{8}$, inicialmente en el contexto de las disposiciones técnico-normativas y jurídicas de 1962 sobre la prevención de la silicosis, en las minas, en los puestos industriales y en otros lugares de trabajo en los que existía riesgo de esta enfermedad profesional $y$, posteriormente, en empresas del sector secundario de actividades económicas de una cierta envergadura?

En 1966 es creada la Escuela Nacional de Salud Pública y de Medicina Tropical ${ }^{10}$ transfiriéndose el referido Curso de Medicina del Trabajo para esta institución y en 1972, es creada a Escuela Nacional de Salud Pública (y el Instituto de Higiene y Medicina Tropical), inicialmente, en las instalaciones del Instituto Nacional de Salud (INSA) y, a partir de 1976, de forma autónoma ${ }^{11}$, año en que dejo de formar parte del sector de enseñanza del INSA.

A partir de 1989, además del curso de la Escuela Nacional de Salud Pública (ENSP), fueron creados dos nuevos cursos de Medicina del Trabajo, el primero de ellos en la Facultad de Medicina de la Universidad de Oporto y el segundo en la Facultad de Medicina de la Universidad de Coimbra ${ }^{7}$, que, tal como el de la ENSP, todavía se mantienen en la actualidad.

El diploma del Curso de Medicina del Trabajo dio competencias suficientes para la práctica profesional hasta 1994 (Decreto-ley 26/94, de 1 de febrero con las alteraciones introducidas por la Ley $\mathrm{n}^{\circ} 7 / 95$, de 29 de marzo y, más tarde, por el Decreto-ley $\mathrm{n}^{\circ}$ 109/2000, de 30 de junio), fecha en que el legislativo decidió exigir, para la práctica legal de la Medicina del Trabajo, la obtención del título de especialista ${ }^{12,13,14}$. De hecho, desde esta fecha se empezó a considerar medico del trabajo “...el licenciado en Medicina con especialidad de Medicina del Trabajo reconocida por la Ordem dos Médicos (el Colegio Oficial de Médicos) ..." (n 2 del art $^{\circ} 25$ del Decreto-ley $n^{\circ}$ 109/2000, de 30 de junio). Señalase a este propósito que en Portugal la creación, en el Colegio Oficial de Médicos, de la especialidad de Medicina del Trabajo se establece a final de los años 70.

El número de médicos del trabajo por 10.000 habitantes es muy variable en diferentes países de Europa ${ }^{15}$, estimándose que en Portugal pueda ser de 1.5 con base en el número de especialistas inscritos en el respectivo colegio de la Especialidad del Colegio Oficial de Médicos $^{16}$ (Figura 4).

Figura 4 - Portugal: estimativa del número de médicos del trabajo por 10000 trabajadores.

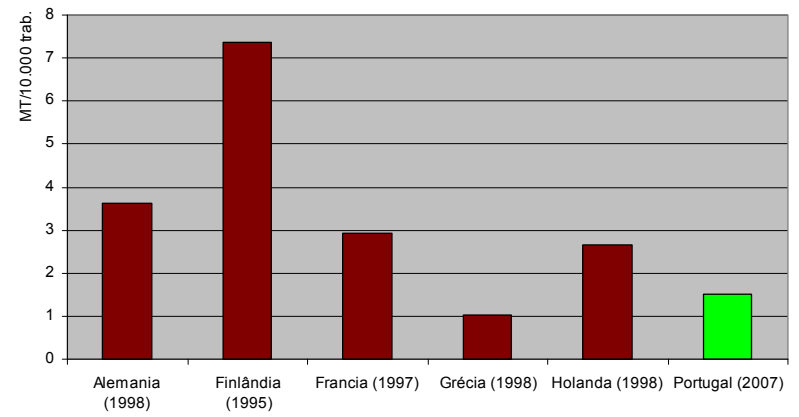

Portugal - Inscritos en el Colegio de Especialidad de Medicina del Trabajo de la Ordem dos Médicos. 


\section{ORGANIZACIÓN DE LOS SERVICIOS DE MEDICINA DEL TRABAJO}

En Portugal los Servicios de Medicina del Trabajo fueron creados (y organizados) a partir de los años 70 en función del riesgo de enfermedad profesional (y en función del tamaño de la empresa) ${ }^{17,18,19,20}$. Por tanto, esta organización sucedió 16 años después de Francia $^{21}$ e 6 años después de España ${ }^{22}$.

Los primeros textos legales de encuadramiento de la prevención de riesgos profesionales datan de 1962 y establecen un conjunto de normativas de organización destinadas a prevenir la silicosis ${ }^{17}, 18$. Comienza a ser obligatorio para las empresas efectuar la medición de los niveles de exposición a la sílice libré y a ser igualmente obligatoria la vigilancia médica de los trabajadores expuestos. Es en el segundo diploma legal ${ }^{18}$, que reglamenta el primero, donde se hace referencia al "medico del trabajo". Cinco años después de la legislación de 1962, los Servicios de Medicina del Trabajo pasan a cubrir también las empresas industriales con más de 200 trabajadores y/o las empresas donde existe riesgo de enfermedad profesional, independientemente del número de empleados.

Esta obligatoriedad de la organización de servicios médicos del trabajo en empresas industriales con más de 200 trabajadores limitó la tasa de cobertura de la población activa empleada a un estimado de 15\%. De hecho, el número de trabajadores cubiertos por los servicios de Medicina del Trabajo era apenas de un $7.2 \%$ en 1971 y de un $7.9 \%$ en $1981^{23}$. Citando los mismos autores, en 1987, la estimativa de la tasa de cobertura de la población activa, publicada por la Dirección General de Cuidados de Salud Primarios (DGCSP), era de $13 \%$ y en $1990^{24}$ era del $13.7 \%$.

La entrada de Portugal para la Comunidad Económica Europea, en 1986, tuvo como consecuencia, en el campo de la Seguridad, Higiene y Salud en el Trabajo (SH\&ST), la modernización del cuadro legal, a través de la transposición, en 1991, de la Directiva 89/391/CEE, de 12 de junio, conocida como Directiva-Marco ${ }^{25,}{ }^{26}$.

En 1994 es publicado el primer diploma reglamentario de la organización y funcionamiento de las actividades de SHST, el Decreto-ley $n^{\circ}$ 26/94 de 1 de febrero ${ }^{12}$, que vino a sustituir la legislación equivalente de la década de los $60^{17,18,19,20}$ y a mejorar la tasa de cobertura de la población activa.

Las actuales disposiciones legales sobre SST $^{30}$ determinan que el empresario debe organizar servicios de Seguridad, Higiene y Salud en el Trabajo con los siguientes objetivos:

- Establecimiento y mantenimiento de condiciones de trabajo que aseguren la integridad física y mental de los trabajadores;

- Desenvolvimiento de condiciones técnicas que aseguren la existencia de buenas condiciones en materia de SH\&ST;

- Información y formación en SH\&ST de los trabajadores;

- Información y consulta de los trabajadores y sus representantes.

Esta organización puede ser realizada de una de las siguientes formas: (a) servicios internos; (b) servicios entre-empresas e (c) servicios externos. El empresario puede incluso optar por modalidades diferentes de organización para cada establecimiento y puede optar por la organización separada de la actividad de Salud, por un lado, y la de Seguridad e Higiene, por otro.

Los servicios internos, que forman parte de la estructura de la empresa y dependen del empresario, son organizados en empresas o establecimientos que desenvuelvan actividades de riesgo elevado y que estén expuestos, por lo menos 30 trabajadores y en empresas con, un mínimo, de 400 trabajadores, independientemente de la actividad realizada. Se consideran actividades de riesgo elevado las siguientes:

- Trabajos en obras de construcción, excavación, movimientos de tierras, túneles, con riesgo de caídas de alturas o de enterramientos, demoliciones e intervenciones en líneas de trenes o vías de automóviles sin interrupción del tráfico; 
- Actividades de industrias extractivas;

- Trabajo hiperbárico;

- Actividades que envuelvan la utilización o almacenamiento de cantidades significativas de productos químicos peligrosos susceptibles de provocar accidentes graves;

- Elaboración, transporte y utilización de explosivos y pirotecnia;

- Actividades de la industria siderúrgica y de la construcción naval;

- Actividades que conlleven contacto con corrientes eléctricas de media y alta tensión;

- Elaboración y transporte de gases comprimidos, liquidificados o disueltos, o a utilización significativa de los mismos;

- Actividades que impliquen la exposición de radiaciones ionizantes;

- Actividades que impliquen la exposición de agentes cancerígenos, mutagénicos o tóxicos para la reproducción;

- Actividades que impliquen la exposición de agentes biológicos del grupo 3 o 4;

- Trabajos que conlleven riesgo de silicosis.

Adicionalmente, la promoción de la vigilancia de la salud puede ser asegurada por el Servicio Nacional de Salud a los trabajadores en las siguientes situaciones:

- Trabajadores autónomos;

- Trabajadores agrícolas de temporada;

- Aprendices de artesanos;

- Trabajadores del servicio domestico;

- Trabajadores de pesca de campaña;

En micro-empresas (máximo 10 trabajadores) con actividades que no sean consideradas de riesgo elevado.

Por comparación al cuadro legal de los años 60, los modelos de organización de servicios, en el actual encuadramiento técnico-normativo permite una amplia flexibilidad de formas organizativas, que se tiene reflejado en una opción mayoritaria en el modelo privado de organización de los servicios de SH\&ST (servicios externos). En 2001 la lista de teléfonos de empresas ofrecía 2006 empresas prestadoras de servicios externos de SH\&ST, tanto en la vertiente "Médicos - medicina del trabajo", como en la vertiente "Seguridad e Higiene en el Trabajo - servicios"31.

Las razones de la actual opción mayoritaria de la organización de servicios estarán, por cierto, relacionadas con la dimensión de nuestras empresas (Tabla I), por la distribución mayoritaria del sector terciario (Tabla II) y, entre otros, por el dinamismo de la iniciativa privada de los empresarios (y técnicos) de SH\&ST.

Tabla II - Distribución de la población activa en Portugal por los tres grandes sectores de actividad económica y de su evolución en los últimos 47 años (1960-2007).

\begin{tabular}{cccc}
\hline AÑo & Sector primário (\%) & Sector secundário (\%) & Sector Terciário (\%) \\
\hline 1960 & 43,6 & 27,1 & 29,3 \\
1970 & 31,7 & 32,3 & 36,0 \\
1981 & 19,3 & 39,1 & 41,6 \\
1991 & 10,8 & 37,9 & 51,3 \\
1996 & 12,2 & 31,4 & 56,4 \\
2007 & 11,8 & 30,6 & 57,6 \\
\hline
\end{tabular}

Fontes: Santos e Uva (en proceso de publicación) e INE, 2008. 
Las grandes empresas con 500 o más trabajadores a su servicio totalizan, de hecho, apenas $0.1 \%$ del universo de las empresas en Portugal empleando, a pesar de esto, cerca del $\mathbf{1 8 . 6 \%}$ de la población laboral ${ }^{32}$. El conjunto de las medianas empresas con 50 a 499 trabajadores corresponden al $2.8 \%$ de las empresas que emplean cerca del $29.8 \%$ de la población activa empleada.

La actual legislación sobre la organización de los servicios de SH\&ST, a pesar de permitir diferentes modalidades de organización de servicios, no es esclarecedora en cuanto a las formas de articulación entre estas actividades, específicamente las centradas en el ambiente del trabajo y las centradas en los trabajadores. Queda, de este modo, perjudicada la integración de gestión de SH\&ST, por ejemplo en materia tan decisiva como el sistema de información de la gestión ${ }^{31}$.

\section{ALGUNOS DATOS SOBRE ACCIDENTES DE TRABAJO Y ENFERMEDADES PROFESIONALES}

Los aspectos relacionados con la Salud y Seguridad del Trabajo envuelven, casi siempre, la referencia a algunos indicadores sobre accidentes de trabajo y enfermedades profesionales, una vez que constituyen dos de las principales consecuencias más negativas en materia de salud y seguridad en el trabajo ${ }^{33}$. Aunque den una visión parcial, y por veces retorcida, de los resultados en materia de prevención de los riegos profesionales, envuelven esencialmente los factores de riesgo "tradicionales", específicamente de naturaleza física, química e (micro)biológica.

\section{Enfermedades profesionales}

Fue publicado, en 1936, las primeras normas de reparación de daños consecuentes de accidentes de trabajo y de enfermedades profesionales ${ }^{34}$ que incluía la primera Lista de Enfermedades Profesionales. Estaban constituida por entonces solamente de siete entidades clínicas: intoxicaciones por (i) el plomo; por (ii) el mercurio; por (iii) colorantes $\mathrm{y}$ disolventes nocivos; por (iv) polvo, gases y vapores industriales; por (v) rayos $\mathrm{X} \mathrm{y}$ substancias radioactivas; y también; (vi) la infección carbunculosa y (vii) las dermatosis profesionales $^{1}$. Posteriormente se siguieron varias actualizaciones de la Lista de Enfermedades Profesionales, la última de ellas realizada en $2007^{35,38,39,40}$. La actual versión de la lista tiene los siguientes cinco capítulos organizados en diferentes cuadros nosológicos:

1. Enfermedades provocadas por agentes químicos, con 31 entidades patológicas;

2. Enfermedades del aparato respiratorio, con 5 entidades;

3. Enfermedades cutáneas y otras, con 22 entidades;

4. Enfermedades provocadas por agentes físicos, con 14 entidades;

5. Enfermedades infecciosas y parasitarias, con 42 entidades;

Existen además los capítulos de $\operatorname{los}^{6}$ tumores $\mathrm{y}^{7}$ de las manifestaciones alérgicas de las mucosas que remiten para entidades nosológicas de los anteriores cinco capítulos.

Cada cuadro, además de la codificación, está compuesto de cuatro columnas: (i) factor (o factores) de riesgo; (ii) enfermedades y otras manifestaciones clínicas; (iii) caracterización (periodo indicativo) solo aplicable a las situaciones en que se verifica suspensión de la exposición al factor de riesgo y (iv) lista ejemplificada (o limitativa en algunos cuadros) de los trabajos susceptibles de provocar la enfermedad.

Los datos estadísticos sobre enfermedades profesionales envuelven las enfermedades comunicadas $^{36}$, cuando envuelven incapacidad permanente o no (Figuras 5 e 6) y afectan, actualmente, mas al sexo femenino y son mayoritariamente causadas por agentes físicos (Figura 7). 
Figura 5 - Portugal: Enfermedades profesionales, por sexo (2001 - 2006).

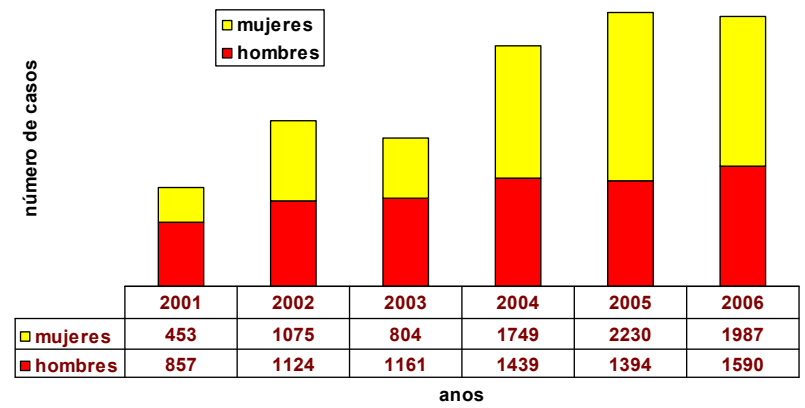

Fonte: MESS - Instituto de Informática. Departamento gestão da informação, 2008

Figura 6 - Portugal: Enfermedades profesionales, por incapacidad (2001 - 2006).

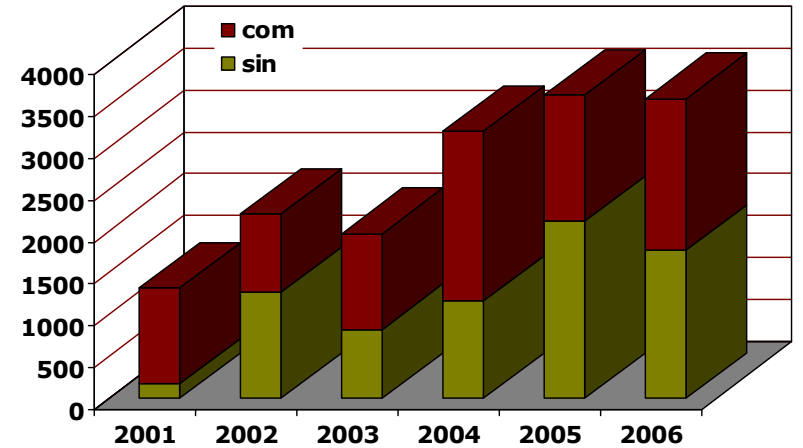

Fonte: MESS - Instituto de Informática. Departamento gestão da informação, 2008.

Figura 7 - Portugal: Enfermedades profesionales, por causas (2001 - 2006).

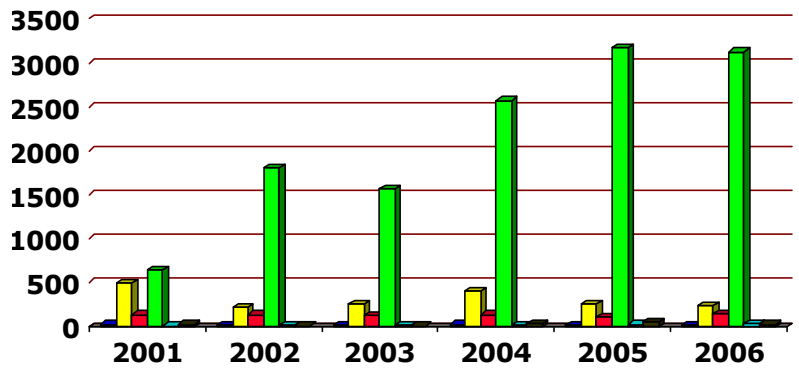

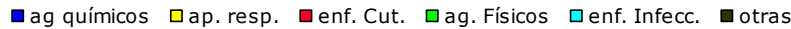

Fonte: MESS - Instituto de Informática. Departamento gestão da informação, 2008.

\section{Accidentes de trabajo}

En términos europeos la tasa de incidencia de accidentes de trabajo tiene venido a disminuir (Figura 8), aun cuando Portugal mantenga tasas de accidentes de trabajo (incluyendo los accidentes mortales) muy elevadas (Figura 9 e 10).

Figura 8 - Europa: Tasa de incidencia de accidentes de trabajo (por 100000 trabajadores).

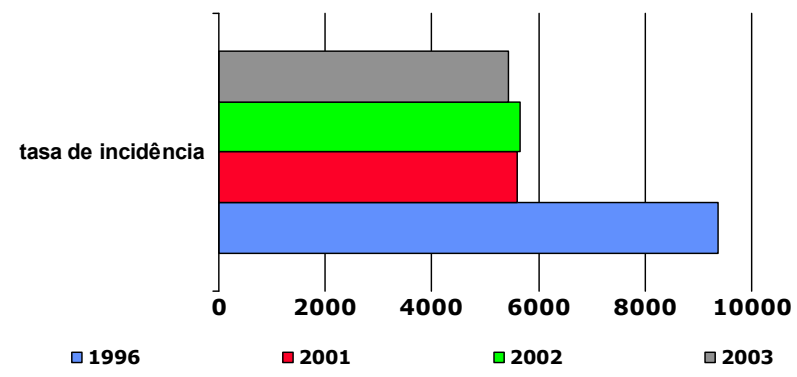

Fonte: Eurostat - European Statistics on accidents at work, 2004. 
Figura 9 - Europa: Accidentes de trabajo (4 o más días de ITA) - "standardized incidence rate" por 100.000 trabajadores.

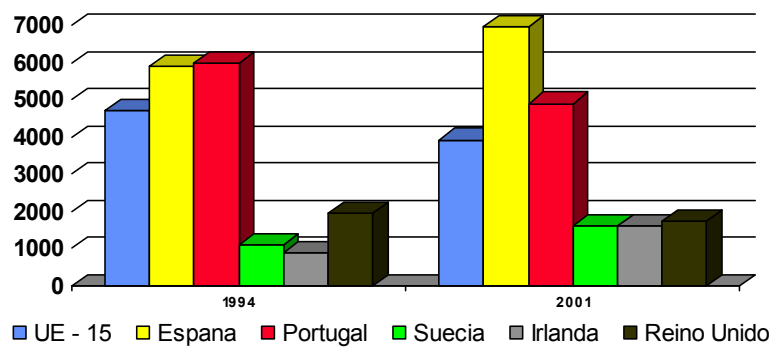

Fonte: Eurostat - European Statistics on accidents at work, 2004.

Figura 10 - Europa: Accidentes de trabajo mortales - "standardized incidence rate" por 100.000 trabajadores.
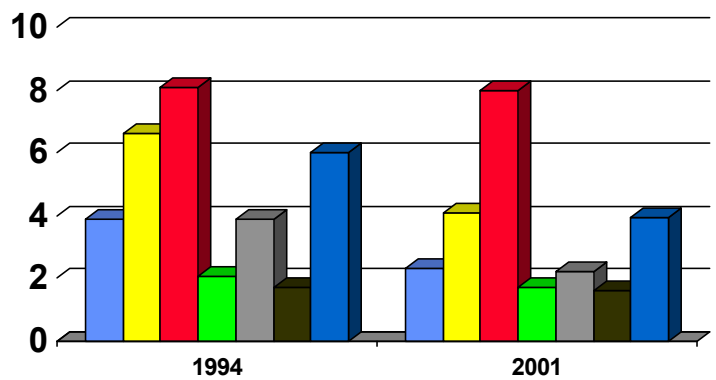

口UE - $15 \square$ Espana $\square$ Portugal (2000) $\square$ Suecia $\square$ Irlanda $\square$ Reino Unido $\square$ Bélgica

Fonte: Eurostat - European Statistics on accidents at work, 2004.

Los accidentes de trabajo mortales en Portugal continúan a tener una importante expresión en el sector de la Construcción. Probablemente relacionado con la población activa empleada en este sector de actividad, en 2007 (Figura 11 e 12), 12 de los 82 accidentes mortales que sucedieron en el sector de la construcción (15\%), ocurrieron en trabajadores inmigrantes de 7 países diferentes ${ }^{37}$. Según la causa de muerte, la caída de altura es la principal razón de causa de accidentes de trabajo mortales (Figura 13).

Figura 11 - Portugal: accidentes de trabajo mortales, por sector de actividad - 2007.
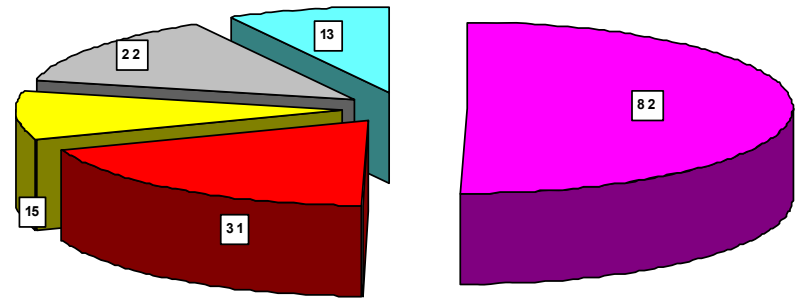

$\square$ C. Civil $\square$ ind. Transformadora $\square$ Agric/Pescas $\square$ Comercio y Servicios $\square$ Otros Fonte: Inspecção Geral do Trabalho, 2008.

Figura 12 - Portugal: accidentes de trabajo mortales (Construcción Civil).

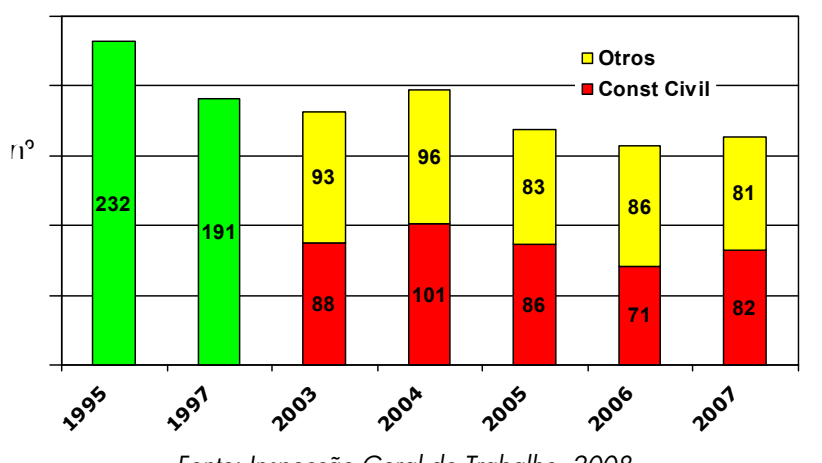

Fonte: Inspecção Geral do Trabalho, 2008 
Figura 13 - Portugal: accidentes de trabajo mortales, según las causas (2007).

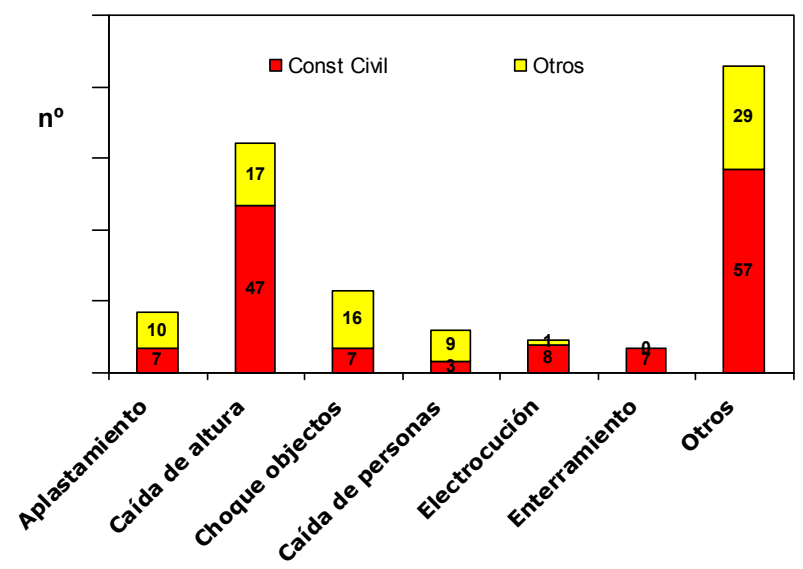

\section{ESTRATEGIA PORTUGUESA PARA LA SEGURIDAD Y SALUD EN EL TRABAJO}

Data de la década de 1990 la firma del primer acuerdo sobre SH\&ST, en el contexto del Acuerdo Económico y Social, subscrito por la Confederación General de los Trabajadores Portugueses (CGTP-IN) y por la Confederación de los Agricultores de Portugal $^{27}$. Posteriormente, en Febrero de 2001, los sindicatos y entidades empresariales firmaban un "Acuerdo sobre las Condiciones del Trabajo, Higiene y Seguridad en el Trabajo y Lucha contra la Siniestralidad" que definía como objetivos estratégicos: (i) La armonización de la modernización de las empresas con la Salud y Seguridad en el Trabajo (SST); (ii) la promoción de la difusión y del fomento de una cultura de prevención de riesgos profesionales y (iii) la disminución de accidentes de trabajo y de enfermedades profesionales $^{28}$.

Además de los objetivos que pretenden movilizar la sociedad y perfeccionar las políticas públicas de SST para la promoción de la salud de los trabajadores y para la prevención de los riesgos profesionales, continúan a valorizarse los aspectos que los servicios de prestación de cuidados tienen en este dominio, particularmente los que se dirigen para las pequeñas y medianas empresas.

La actual estrategia nacional para la Seguridad y Salud en el Trabajo (2008-2012) fue recientemente aprobada (Resolución del Consejo de Ministros $n^{\circ}$ 59/2008, del 12 de marzo ${ }^{29}$, en el contexto de la estrategia europea 2007-2012, y define los siguientes dos ejes fundamentales (1): Desenvolvimiento de políticas públicas coherentes y eficaces a través de la colaboración entre los diferentes Departamentos de Administración Pública y (2) promoción de la seguridad y salud en los puestos de trabajo como presupuesto de una mejoría efectiva de las condiciones de trabajo. En el primer punto se establecen 7 objetivos:

(i) Desenvolver una cultura de prevención;

(ii) Mejorar el sistema de información sobre SST;

(iii) Incluir la SST en los sistemas educativos y de investigación;

(iv) Dinamizar el Sistema Nacional de Prevención de Riesgos Profesionales;

(v) Mejorar la coordinación de los servicios públicos en el área de SH\&ST;

(vi) Promulgar, perfeccionar y simplificar las normas de SST y

(vii) Establecer el modelo de la Autoridad para las Condiciones del Trabajo;

y en el segundo punto, tres objetivos:

(viii) Promover el cumplimiento efectivo de la legislación de Sh\&ST, en especial en las pequeñas empresas; 
(ix) Mejorar la calidad de prestación de servicios de SH\&ST y aumentar las competencias de sus profesionales y

(x) Profundizar en el papel de los sindicatos y entidades empresariales e envolver a los empresarios y trabajadores en la mejoría de las condiciones de trabajo.

Las políticas de Salud y Seguridad del Trabajo (entiéndase "policy" y no "politics") se integran dentro de los derechos fundamentales, tales como el derecho a la vida y a la integridad física, el derecho al trabajo o, incluso, el derecho a la salud. Las respectivas estrategias constituyen "instrumentos" indispensables para, a partir de un determinado diagnostico, establecer objetivos y metas "evaluables" ("inteligentes") en el periodo al que se definen.

Cualquier estrategia nacional de Salud y Seguridad en el Trabajo, o mismo solamente de prevención de riscos profesionales, debe partir de un correcto diagnostico de la situación que identifique los aspectos menos positivos de las políticas hasta entonces seguidas. En este contexto se debe asumir que, en Portugal, se observaron desenvolvimientos importantes en el área de la Salud y Seguridad del Trabajo desde mediados de la década de 1990, así como todavía se mantienen problemas que pueden estar en el origen de alguna dificultad en la aplicación de políticas de promoción de la salud en el trabajo y de la prevención de los riesgos profesionales. En este contexto, pretendería destacar las siguientes:

1. La coordinación intersectorial de la Administración Pública tiene sido insuficiente, principalmente en las áreas de Trabajo y Salud, lo que no tiene promocionado las mejores políticas activas que, de hecho, potencien una cultura de salud y seguridad del trabajo o, incluso de prevención de los riesgos profesionales;

2. Las políticas de Seguridad y Salud de los trabajadores en los puestos de trabajo tiene sido, casi exclusivamente, basadas en la promulgación de diplomas legales (o normativos) y menos en el acompañamiento y control de su aplicación en un país caracterizado, como se refirió, por una estructura empresarial de pequeñas empresas, o incluso micro-empresas;

3. Tiene sido, actualmente, atribuida una mayor atención a la prevención ambiental de los riesgos profesionales en perjuicio de la vigilancia de la salud de los trabajadores. Dicho de otra forma, no tiene sido valorizada la evaluación "individual" de los riesgos profesionales que constituye, de hecho, uno de los principales objetivos de la prevención de los riesgos profesionales;

4. Finalmente, la promoción de la salud de los trabajadores, mismo en la perspectiva más "clásica" basada en los determinantes de la salud, tiene sido sistemáticamente desvalorizada y, en su componente "generadora de salud", casi totalmente despedazada, designadamente en los componentes de "conforto" o de "bien-estar" que el trabajo debería conllevar.

La evolución (reciente) de las políticas de salud y seguridad asociada a la "capacidad" de los empresarios y los empleados auguran que la respectiva documentación en SST, basada en mejores percepciones de los riesgos profesionales y la mayor valorización de la salud como valor positivo, conducirán, inevitablemente, a puestos de trabajo más seguros y saludables. Ojala que también con trabajadores más seguros y más saludables.

De hecho, los resultados en SST deben ser medidos a través de los beneficios en Salud y Seguridad que constituyen, por cierto, los grandes objetivos de cualquier política y estrategia de acción y no pueden continuar circunscrito a los indicadores de accidentes de trabajo.

Solo una opinión pública que valorice la promoción de los aspectos relativos a la calidad de las condiciones de trabajo en la perspectiva de la Salud y Seguridad del Trabajo podrá determinar los cambios en el mundo del trabajo que puedan contribuir para trabajadores activos más productivos en lugares y ambientes de trabajo más salubres, más seguros e satisfactoriamente más confortables. 


\section{BIBLIOGRAFÍA}

1. Santos C, Uva A. Saúde e segurança do trabalho: notas historiográficas com futuro. Lisboa: Autoridade para as Condições do Trabalho - ACT. en proceso de publicación 2009.

2. INE. Estatísticas do Emprego: $3^{\circ}$ trimestre de 2007. Destaque. [serial on the Internet] 16 de Novembro de 2007. [cited 2008 May 30]; 1-7. Available from: http://www.ine.pt/ngt_server/attachfileu.jsp?look_ parentBoui=9273494\&att_display=n\&att_download $=\mathrm{y}$

3. OSHA. Agência Europeia para a Segurança e Saúde no Trabalho. Segurança e saúde no trabalho: estatísticas. [homepage on the Internet]. [updated 2005 May 11; cited 2008 May 31]. Lisboa: Agência Europeia para a Segurança e Saúde no Trabalho; 1997. [about 12 screens]. Available from: http://pt.osha. europa.eu/statistics/static.stm.

4. IAPMEI. Sobre as PME: PME na estrutura empresarial nacional. [homepage on the Internet]. [updated 2008 July 26; cited 2008 May 29]. Lisboa: Instituto de Apoio às Pequenas e Médias Empresas e ao Investimento. Ministério da Economia e da Inovação; 2008. Available from: http://www.iapmei.pt/iapmei-faq-02. php?tema=7.

5. ISHST. Os serviços nacionais de prevenção não estão paralisados. Comunicado à Imprensa. [serial on the Internet] 19 de Setembro de 2005. [cited 2006 October 10]. Lisboa: Instituto para a Segurança, Higiene e Saúde no Trabalho; 2005. Available from: http://www.ishst.pt/ISHST_NoticiaPress.aspx?nid=A000000000003364

6. ISHST. ISHST 2005: um ano de actividade. [powerpoint on the Internet]. Lisboa: Instituto para a Segurança, Higiene e Saúde no Trabalho; 2008 [cited 2008 May 13]. Available from: http://www.ishst.pt/downloads/ content/síntese_relatório2005.pdf .

7. Uva A. Medicina do trabalho: patologia e clínica: relatório da disciplina: programa, conteúdos e métodos de ensino. Lisboa: Escola Nacional de Saúde Pública. Universidade Nova de Lisboa; 2002.

8. Decreto-Lei $\mathrm{n}^{\circ}$ 44308. DR Série I. 95 (1962-04-27) 577-578. Insere disposições destinadas a promover a prevenção médica da silicose.

9. Melo FG, Faria JGS, Reis V, Uva AS. Escola Nacional de Saúde Pública: abertura do ano escolar 1999-2000. Revista Portuguesa de Saúde Pública. 2000; 18(1): 21-25.

10. Decreto-Lei $n^{\circ}$ 47102. DR Série I. 164 (1966-07-16) 1268-1272. Extingue o Instituto de Medicina Tropical e cria em Lisboa e na dependência dos Ministérios do Ultramar e da Saúde e Assistência a Escola Nacional de Saúde Pública e de Medicina Tropical.

11. Decreto-Lei no 278/76. DR Série I. 89 (1976-04-14) 815-816. Separa a Escola Nacional de Saúde Pública do Instituto Nacional de Saúde, deixando de constituir o sector de ensino deste Instituto.

12. Decreto-Lei $n^{\circ}$ 26/94. DR Série I-A. 26 (1994-02-01) 480-486. Estabelece o regime de organização e funcionamento das actividades de segurança, higiene e saúde no trabalho.

13. Lei ${ }^{\circ}$ 7/95. DR Série I-A. 75 (1995-03-29) 1710-1713. Alteração, por ratificação, do Decreto-Lei n. ${ }^{\circ}$ 26/94, de 1 de Fevereiro.

14. Decreto-Lei $n^{\circ}$ n. ${ }^{\circ}$ 109/00. DR Série I - A. 149 (2000-06-30) 2835-2847. Altera o Decreto-Lei n. ${ }^{\circ}$ 26/94, de 1 de Fevereiro, alterado pelas Leis $n^{\circ}$ s 7/95, de 29 de Março, e 118/99, de 11 de Agosto, que contém o regime de organização e funcionamento das actividades de segurança, higiene e saúde no trabalho.

15. Graça L. Política(s) de saúde no trabalho: um inquérito sociológico às empresas portuguesas. Lisboa: Escola Nacional de Saúde Pública. Universidade Nova de Lisboa, 2004. Tese de doutoramento em Saúde Pública, especialização em Saúde Ocupacional, pela UNL.

16. Ordem dos Médicos. Distribuição por especialidade, idade e sexo 2007. Lisboa: Portal Oficial da Ordem dos Médicos. [updated 2007; cited 2008 June 17]. Available from: http://www.ordemdosmedicos.pt/?lop=stats_ medicos\&type $=1 \&$ ano $=2007$

17. Decreto-Lei $\mathrm{n}^{\circ}$ 44308. DG Série I. 95 (1962-04-27) 577-578. Insere disposições destinadas a promover a prevenção médica da silicose.

18. Decreto-Lei no 44537. DG Série I. 192 (1962-08-22). 1154-1156. Regula a organização dos serviços médicos do trabalho para a prevenção médica da silicose, referida no Decreto-Lei n. ${ }^{\circ} 44308$.

19. Decreto-Lei $\mathrm{n}^{\circ}$ 47511. DG I Série. 21 (1967-01-25) 125-126. Determina que nas empresas industriais e comerciais sejam organizados serviços médicos de trabalho.

20. Decreto-Lei $n^{\circ}$ 47512. DG I Série. 21 (1967-01-25) 126-128. Promulga o Regulamento dos Serviços Médicos do Trabalho das Empresas.

21. France. Président de la République. Message du Président de la République à l' occasion du cinquantenaire de la médecine du travail le 10 octobre 1996. Paris: Archives de la Présidence de M. Jacques Chirac 19952007. [homepage on the Internet]. [cited 2006 December 06]. Available from: http://www.elysee.fr/elysee/ 
francais/interventions/lettres_et_messages/1996/octobre/message_du_president_de_la_republique_a_1_ occasion_du_cinquantenaire_de_la_medecine_du_travail.1268.html.

22. Sociedad Española de Medicina y Seguridad del Trabajo. Madrid: Sociedad Española de Medicina y Seguridad del Trabajo. [updated 2008 July 21]; cited 2006]. Available from: http://www.semst.org/.

23. Faria M, Santos C S. Alguns dados estatísticos com interesse em saúde ocupacional. Paço de Arcos: Edição SO; 1989.

24. Ministério da Saúde. Direcção-Geral dos Cuidados de Saúde Primários. Relatório anual de saúde ocupacional. Lisboa: Direç̧ão de Serviços de Saúde Escolar e Ocupacional; 1991.

25. Decreto-Lei $\mathrm{n}^{\circ}$ 441/91. DR Série I-A. 262 (1991-11-14) 5826-5833. Estabelece o regime jurídico do enquadramento da segurança, higiene e saúde no trabalho.

26. Decreto-Lei no 133/99. DR Série I-A. 93 (1999-04-21) 2117-2119. Altera o Decreto-Lei n. ${ }^{\circ}$ 441/91, de 14 de Novembro, relativo aos princípios da prevenção de riscos profissionais, para assegurar a transposição de algumas regras da directiva quadro relativa à segurança e saúde dos trabalhadores nos locais de trabalho.

27. Conselho Permanente de Concertação Social. Acordo Económico e Social, Lisboa: CPCS; Julho de 1991.

28. Uva A - Formação em saúde e segurança do trabalho: aonde ir ou quo vadis? In: VI Congresso Nacional de Saúde Ocupacional, Póvoa do Varzim, 2 a 4 de Novembro de 2006: Conferência plenária de abertura. Porto: Faculdade de Medicina do Porto; 2006.

29. Resolução do Conselho de Ministros n 59/2008. DR Série I. 64 (2008-04-01) 1984-1995. Aprova a Estratégia Nacional para a Segurança e Saúde no Trabalho, para o período 2008-2012.

30. Lei $n^{\circ}$ 35/2004, DR Série I-A. 177 (2004-07-29) 4810-4885. Regulamenta a Lei no 99/2003, de 27 de Agosto, que aprovou o Código do Trabalho.

31. Graça L, Uva AS. Saúde e segurança do trabalho: da lógica do serviço ao sistema integrado de gestão. Saúde \& Trabalho. 2007; 6: 119-144.

32. INE. In 2006, the Economy has grown at different rates in the Portuguese regions: 1995-2006. Press Releases. [serial on the Internet] 28 July 2008. [cited 2008 May 30]. Available from:http://www.ine.pt/portal/page/ portal/PORTAL_INE/Publicacoes?PUBLICACOESpub_boui=5600892\&PUBLICACOESmodo=2.

33. Faria, M.; Uva, A.S. - Diagnóstico e prevenção das doenças profissionais: algumas reflexões. Jornal das Ciências Médicas de Lisboa. 1988; CLII (9,10): 1-12.

34. Lei $\mathrm{n}^{\circ}$ 1942. DG Série I.174 (1936-07-27) 87-92. Regime jurídico dos acidentes de trabalho e doenças profissionais.

35. Decreto Regulamentar n. ${ }^{\circ}$ 76/2007. DR Série I. 136 (2007-07-17) 4499-4543. Altera o Decreto Regulamentar n. ${ }^{\circ}$ 6/2001, de 5 de Maio, que aprova a lista das doenças profissionais e o respectivo índice codificado.

36. MTSS. Instituto de Informática, IP. Estatísticas disponíveis: doenças profissionais. Lisboa: Instituto de Informática, IP.; 2008. [updated 2008; cited 2008 June 21]. Available from: http://195.245.197.202/left. asp?02.21.03.07.

37. Inspecção Geral do Trabalho. Autoridade para as Condições de Trabalho - ACT. Acidentes de trabalho mortais objecto de inquérito: informação actualizada a 19 de Julho de 2008. Lisboa: Autoridade para as Condições de Trabalho - ACT, 2008. [cited 2008 June 17]. Available from: http://www.igt.gov.pt/DownLoads/ content/Estatisticas_Acidentes_Mortais_ACT_2004_2008.pdf.

38. Decreto Regulamentar $n^{\circ}$ 12/80. DR I Série. 106 (1980-05-08) 907-924. Procede à revisão da lista das doenças profissionais actualmente em vigor.

39. Despacho Normativo ${ }^{\circ}$ 253/82. DR I Série. 270 (1982-11-22) 3896-3901. Actualiza as listas de doenças profissionais.

40. Decreto Regulamentar no 6/2001. DR Série I-B. 104 (2001-05-05) 2613-2638. Aprova a lista das doenças profissionais e o respectivo índice codificado.

\section{AGRADECIMIENTO}

Al colega Javier Heranz, médico del trabajo, amigo y alumno. 